\title{
The No-Ellipsoidal Bound of Reachable Sets for Neutral Markovian Jump Systems with Disturbances
}

\author{
Changchun Shen, Shouwei Zhou, Hongyong Deng \\ College of Data Science and Information Engineering, Guizhou Minzu University, Guiyang, China \\ Email: sccyjs2008@163.com, zswyjs_2018@163.com,dhycel@126.com
}

How to cite this paper: Shen, C.C. Zhou, S.W. and Deng, H.Y. (2020) The No-Ellipsoidal Bound of Reachable Sets for Neutral Markovian Jump Systems with Disturbances. Journal of Applied Mathematics and Physics, 8, 799-813. https://doi.org/10.4236/jamp.2020.85062

Received: April 7, 2020

Accepted: May 5, 2020

Published: May 8, 2020

Copyright $\odot 2020$ by author(s) and Scientific Research Publishing Inc. This work is licensed under the Creative Commons Attribution International License (CC BY 4.0).

http://creativecommons.org/licenses/by/4.0/

\begin{abstract}
This paper is concerned with the reachable set estimation problem for neutral Markovian jump systems with bounded peak disturbances, which was rarely proposed for neutral Markovian jump systems. The main consideration is to find a proper method to obtain the no-ellipsoidal bound of the reachable set for neutral Markovian jump system as small as possible. By applying Lyapunov functional method, some derived conditions are obtained in the form of matrix inequalities. Finally, numerical examples are presented to demonstrate the effectiveness of the theoretical results.
\end{abstract}

\section{Keywords}

Reachable Set, No-Ellipsoidal Bound, Linear Neutral System, Lyapunov-Krasovskii, Linear Matrix Inequalities

\section{Introduction}

In practice and engineering applications, many dynamical systems may cause abrupt variations in their structure, due to stochastic failures or repairs of the components, changes in the interconnections of subsystems, sudden environment changes, and so on. Markovian jump systems, modeled by a set of subsystems with transitions among the models determined by a Markov chain taking values in a finite set, have appealed to a lot of researchers in the control community. In the past few decades, the Markovian jump systems have been extensively studied, see [1] [2] [3] [4] and the references therein.

The reachable set [5] estimation of dynamic systems is to derive some closed bounded set that bounds the state trajectories starting from the origin by inputs with peak value. Reachable set estimation is not only an important issue in the 
control theory [6] [7] [8] [9], which plays an important role in solving the problem of state estimation and parameter estimation, but also in practical engineering when safe operation is required through synthesizing controllers to avoid undesirable (or unsafe) regions in the state space. For the bound of reachable sets for linear systems without any time delay, we can find a well-known result which has been formulated in terms of linear matrix inequality (LMI) [10], and it is widely used to design control systems that have saturating actuators [11] [12]. However, time delay phenomenon is frequently encountered in many practical systems, such as biological systems, chemical systems, hydraulic systems and electrical networks. It is well known that the existence of time delays in a system may cause instability or bad system performance (see [13] [14] [15]). Hence it is natural to ask what about the reachable set of systems with time-delays.

Recently, the reachable set estimation problems for time-delay systems have been received growing attention. Then, an increasing number of researchers have devoted their efforts to the problem of reachable set estimation [16] [17] [18]. In [16], a delay-dependent condition for an ellipsoid bounding the set of reachable states was presented by using the Lyapunov-Razumikhin function and the S-procedure. Five non-convex scalar parameters have to be treated as tuning parameters to find the "smallest" possible ellipsoid. Recently, Kim [17] modified the Lyapunov-Krasovskii functional used for exponential stability analysis [19] [20], and, based on it, he proposed an improved ellipsoidal bound of the reachable set of reachable states. The derivative of the time delay is assumed to be less than one in [16]. This limitation may restrict the scope of application for this method. However, if the value of the derivative of the time delay is large, this method will yield a bigger ellipsoid bounding the reachable set than that in [16]. The reachable set bounding for delayed systems subject to both polytopic uncertainties and bounded peak inputs was considered in [21]. Some criteria bounding the reachable set are derived, by using a maximal Lyapunov-Krasovskii functional, which is constructed by taking pointwise maximum over a family of Lyapunov-Krasovskii functionals. But the bound of reachable set bounding for delayed systems with polytopic uncertainties is not small enough.

As is well known, neutral system being a special case of time delay system exists in many dynamic systems [22] [23] [24] [25]. However, the bound of reachable sets for neutral Markovian jump systems with bounded peak disturbances has not been investigated, which motivates this paper. In this paper, we consider the problem of finding the no-ellipsoidal bound of reachable sets for neutral Markovian jump systems with bounded peak disturbances. Based on the modified Lyapunov-Krasovskii type functional, some delay-dependent results are derived in the form of matrix inequalities containing only one non-convex scalar. Furthermore, a modified matrix inequality is used to remove the limitation on the variation rate of the delay and obtain a "smaller" no-ellipsoidal bound of reachable sets. Numerical examples illustrate the effectiveness and improvement of the obtained results. 


\section{Problem Statement}

Consider the following neutral Markovian jump systems with disturbances

$$
\begin{aligned}
& \dot{x}(t)-C_{\left(t, r_{t}\right)} \dot{x}(t-\tau)=A_{\left(t, r_{t}\right)} x(t)+B_{\left(t, r_{t}\right)} x(t-h(t))+D_{\left(t, r_{t}\right)} w(t), \\
& x\left(t_{0}+\theta\right)=\varphi(\theta), \quad \forall \theta \in\left[-\rho^{*}, 0\right],
\end{aligned}
$$

where $x(t) \in \mathfrak{R}^{n}$ is the state vector, $\tau>0$ is a constant neutral delay, the discrete delay $h(t)$ is a time-varying function that satisfies

$$
0 \leq h(t) \leq h_{M}, \dot{h}(t) \leq h_{d}<1, w^{\mathrm{T}}(t) w(t) \leq w_{m}^{2},
$$

$\varphi(\cdot)$ is a differentiable vector-valued initial function, $\rho^{*}=\tau+h_{M},\left\{r_{t}, t \geq 0\right\}$ is Markovian process taking values on the probability space in a finite state $\wp=\{1,2,3, \cdots, N\}$ with generator $\Lambda=\left(\lambda_{i j}\right)(i, j \in \xi)$, and $\Lambda$ is described as follows

$$
P\left(r_{t+\Delta}=j \mid r_{t}=i\right)=\left\{\begin{array}{l}
\lambda_{i j} \Delta+o(\Delta), i \neq j \\
1+\lambda_{i i} \Delta+o(\Delta), i=j
\end{array}\right.
$$

where $\Delta>0, \lim _{\Delta \rightarrow+0} \frac{o(\Delta)}{\Delta}=0, \lambda_{i j} \geq 0(i \neq j)$ is the transition probability from $i$ to $j$ at time $t \rightarrow t+\Delta, \quad \lambda_{i i}=-\sum_{j=1, j \neq i}^{N} \lambda_{i j} . A_{\left(t, r_{t}\right)}, B_{\left(t, r_{t}\right)}, C_{\left(t, r_{t}\right)}$ and $D_{\left(t, r_{t}\right)}$ are known constant matrices of the Markov process.

Since the state transition probability of the Markovian jump process is considered in this paper is partially known, the transition probability matrix of Markovian jumping process $\Lambda$ is defined as

$$
\Lambda=\left[\begin{array}{cccc}
\lambda_{11} & ? & \cdots & \lambda_{1 N} \\
? & \lambda_{22} & \cdots & \lambda_{2 N} \\
\vdots & \vdots & \ddots & \vdots \\
\lambda_{41} & ? & \cdots & \lambda_{N N}
\end{array}\right] \text {, }
$$

where ? represents the unknown transition rate. For notational clarity, $\forall i \in \wp$, the set $U^{i}$ denotes $U^{i}=U_{k}^{i} \cup U_{u k}^{i}$ with

$$
\begin{aligned}
& U_{k}^{i}:=\left\{j: \lambda_{i j} \text { is known for } j \in \wp\right\}, \\
& U_{u k}^{i}:=\left\{j: \lambda_{i j} \text { is unknown for } j \in \wp\right\} .
\end{aligned}
$$

moreover, if $U_{k}^{i} \neq \varnothing$, it is further described as $U_{k}^{i}=\left\{k_{1}^{i}, k_{2}^{i}, \cdots, k_{m}^{i}\right\}$, where $m$ is a non-negation integer with $1 \leq m \leq N$ and $k_{j}^{i} \in Z^{+}, 1 \leq k_{j}^{i} \leq N, j=1,2, \cdots, m$ represent the known element of the ith row and jth column in the state transition probability matrix $\Lambda$.

For the sake of brevity, $x(t)$ is used to represent the solution of the system under initial conditions $x\left(t, t_{0}, r_{0}\right)$, and $\{x(t), t\}$ satisfies the initial condition $\left(x_{0}, r_{0}\right)$. And its weak infinitesimal generator, acting on function $V$, is defined in $[3]$.

$$
L V\left(x_{t}, t, i\right)=\lim _{\Delta \rightarrow 0^{+}} \frac{1}{\Delta}\left[E\left(V\left(x_{t+\Delta}, t+\Delta, r_{t+\Delta}\right) \mid\left(x_{t}, r_{t}=i\right)\right)-V\left(x_{t}, t, i\right)\right] .
$$


This paper aims to find a reachable set for neutral Markovian jump systems (1) based on the Lyapunov-Krasovskii functional approach. We denote the set of reachable states with $w(t)$ that satisfies (2) by

$$
\mathfrak{R}_{x} \triangleq\left\{x(t) \in \mathfrak{R}^{n} \mid x(t), w(t) \text { satisfy }(1) \text { and }(2)\right\} .
$$

We will bound $\mathfrak{R}_{x}$ by an ellipsoid of the form

$$
\mathfrak{I}(P, 1) \triangleq\left\{x(t) \in \mathfrak{R}^{n}: x^{\mathrm{T}}(t) P x(t) \leq 1, P>0\right\} .
$$

For simplicity, there are the following representations:

$$
A_{i}=A_{\left(t, r_{t}=i\right)}, B_{i}=B_{\left(t, r_{t}=i\right)}, C_{i}=C_{\left(t, r_{t}=i\right)}, D_{i}=D_{\left(t, r_{t}=i\right)}, P_{i}=P_{\left(t, r_{t}=i\right)} .
$$

In this paper, the following Lemma and Assumption are needed:

Lemma 1 [10]. Let $V(x(0))=0$ and $w^{\mathrm{T}}(t) w(t) \leq w_{m}^{2}$. If

$$
\dot{V}\left(x_{t}\right)+\alpha V\left(x_{t}\right)-\beta w(t)^{\mathrm{T}} w(t) \leq 0, \alpha>0, \beta>0,
$$

then we have $V\left(x_{t}\right) \leq \frac{\beta}{\alpha} w_{m}^{2}$ for $\forall t \geq 0$.

Lemma 2 [4]. Suppose $h \in R^{n}$ and $x(t) \in R^{n}$, for any positive definite matrix $W$ the following inequality holds

$$
-h \int_{t-h}^{t} \dot{x}^{\mathrm{T}}(s) W \dot{x}(s) \mathrm{d} s \leq\left[\begin{array}{ll}
x^{\mathrm{T}}(t) & x^{\mathrm{T}}(t-h)
\end{array}\right]\left[\begin{array}{cc}
-W & W \\
W & -W
\end{array}\right]\left[\begin{array}{c}
x(t) \\
x(t-h)
\end{array}\right] .
$$

Lemma 3 [17]. For any positive-definite matrix $\Phi \in \mathfrak{R}^{n \times n}$, scalar $\gamma>0$, vector function $\omega:[0, \gamma] \rightarrow \mathfrak{R}^{n}$ such that the integrations concerned are well defined, then

$$
\left(\int_{0}^{\gamma} \omega(s) \mathrm{d} s\right)^{\mathrm{T}} \Phi\left(\int_{0}^{\gamma} \omega(s) \mathrm{d} s\right) \leq \gamma \int_{0}^{\gamma} \omega^{\mathrm{T}}(s) \Phi \omega(s) \mathrm{d} s
$$

\section{Main Results}

Our aim is to find an ellipsoid set as small as possible to bound the reachable set defined in (3). In this section, based on an appropriate Lyapunov functional and matrix inequality techniques (Lemma (1-3)), following Theorems are derived.

Theorem 1. Consider the Markov neutral system (1) with constraints (2), if there exist symmetric matrices $P_{2 i}, P_{3 i}, P_{1 i}>0, W_{i}>0 \quad(i=1,2, \cdots, N)$, $Q>0, R>0, S>0$ and a scalar $\alpha>0$ satisfying the following matrix inequalities:

$$
\left[\begin{array}{ccccccc}
\Phi_{i 11} & \Phi_{i 12} & \Phi_{i 13} & P_{2 i}^{\mathrm{T}} C_{i} & 0 & 0 & P_{2 i}^{\mathrm{T}} D_{i} \\
* & \Phi_{i 22} & \Phi_{i 23} & P_{3 i}^{\mathrm{T}} C_{i} & 0 & 0 & P_{3 i}^{\mathrm{T}} D_{i} \\
* & * & \Phi_{i 33} & 0 & \Phi_{i 35} & 0 & 0 \\
* & * & * & -\mathrm{e}^{-\alpha \tau} R & 0 & 0 & 0 \\
* & * & * & * & \Phi_{i 55} & 0 & 0 \\
* & * & * & * & * & \Phi_{i 66} & 0 \\
* & * & * & * & * & * & -\frac{\alpha}{w_{m}} I
\end{array}\right]<0,
$$




$$
\begin{aligned}
& P_{1 i}-W_{i} \leq 0, i \in U_{u k}, i \neq j, \\
& P_{1 i}-W_{i} \leq 0, i \in U_{u k}, i=j,
\end{aligned}
$$

where

$$
\begin{aligned}
& \Phi_{i 11}=P_{2 i}^{\mathrm{T}} A_{i}+A_{i}^{\mathrm{T}} P_{2 i}+Q+\alpha P_{1 i}+\sum_{j \in U_{k}}^{N} \lambda_{i j}\left(P_{1 j}-W_{j}\right), \\
& \Phi_{i 12}=P_{1 i}-P_{2 i}^{\mathrm{T}}+A_{i}^{\mathrm{T}} P_{3 i}, \\
& \Phi_{i 13}=P_{2 i}^{\mathrm{T}} B_{i}, \\
& \Phi_{i 23}=P_{3 i}^{\mathrm{T}} B_{i}, \\
& \Phi_{i 22}=R+\tau h_{M}\left(h_{M}+\tau\right) S-P_{3 i}-P_{3 i}^{\mathrm{T}}, \\
& \Phi_{i 33}=-\left(1-h_{d}\right) \mathrm{e}^{-\alpha h_{M}} Q-h_{M} \mathrm{e}^{-\alpha\left(h_{M}+\tau\right)} S, \\
& \Phi_{i 35}=h_{M} \mathrm{e}^{-\alpha\left(h_{M}+\tau\right)} S, \\
& \Phi_{i 55}=-h_{M} \mathrm{e}^{-\alpha\left(h_{M}+\tau\right)} S, \\
& \Phi_{i 66}=-\tau \mathrm{e}^{-\alpha\left(h_{M}+\tau\right)} S .
\end{aligned}
$$

Then, the reachable set of the system (1) having the constraints (2) is bounded by a non-ellipsoid boundary $\bigcap_{i \in \mathfrak{\wp}} \mathfrak{J}\left(P_{1 i}, 1\right)$, which $\mathfrak{J}\left(P_{1 i}\right)(i \in \wp)$ is defined in (7).

Proof. We choose the following Lyapunov-Krasovskii functional candidate as follows:

$$
V\left(x_{t}, t, r_{t}\right)=\sum_{i=1}^{4} V_{i}\left(x_{t}, t, r_{t}\right)
$$

where

$$
\begin{gathered}
V_{1}\left(x_{t}, t, r_{t}\right)=x^{\mathrm{T}}(t) P_{1 r_{t}} x(t) \\
=\left[\begin{array}{ll}
x^{\mathrm{T}}(t) & \dot{x}^{\mathrm{T}}(t)
\end{array}\right]\left[\begin{array}{ll}
I & 0 \\
0 & 0
\end{array}\right]\left[\begin{array}{cc}
P_{1 r_{t}} & 0 \\
P_{2 r_{t}} & P_{3 r_{t}}
\end{array}\right]\left[\begin{array}{l}
x(t) \\
\dot{x}(t)
\end{array}\right], \\
V_{2}\left(x_{t}, t, r_{t}\right)=\int_{t-h(t)}^{t} \mathrm{e}^{\alpha(s-t)} x^{\mathrm{T}}(s) Q x(s) \mathrm{d} s, \\
V_{3}\left(x_{t}, t, r_{t}\right)=\int_{t-\tau}^{t} \mathrm{e}^{\alpha(s-t)} \dot{x}^{\mathrm{T}}(s) R \dot{x}(s) \mathrm{d} s, \\
V_{4}\left(x_{t}, t, r_{t}\right)=\tau h_{M} \int_{-h_{M}-\tau}^{0} \int_{t+\theta}^{t} \mathrm{e}^{\alpha(s-t)} \dot{x}^{\mathrm{T}}(s) S \dot{x}(s) \mathrm{d} s \mathrm{~d} \theta,
\end{gathered}
$$

where $r_{t} \in \wp, P_{2 i}, \quad P_{3 i}, P_{1 i}>0 \quad(i=1,2, \cdots, N), \quad Q>0, \quad R>0, \quad S>0$ and $\alpha>0$ are solutions of (10).

First, we show that $V\left(x_{t}\right)$ in (11) is a good L-K functional candidate. For $t-h_{M} \leq s \leq t$, we have $0<\mathrm{e}^{-\alpha h_{M}} \leq \mathrm{e}^{\alpha(s-t)} \leq 1$. Furthermore, for $t-\tau \leq s \leq t$, we have $\sum_{i=2}^{4} V_{i}\left(t, x_{t}, r_{t}\right) \geq 0$.

Therefore, we get 


$$
\left\{\begin{array}{l}
V\left(x_{t}, t, r_{t}\right)=\sum_{j=1}^{4} V_{j} \geq V_{1}\left(x_{t}, t, r_{t}\right)=x^{\mathrm{T}}(t) P_{1 r_{t}} x(t), \\
V\left(x_{t}\right)=0, \text { when } x(\theta)=0, \theta \in\left[t-\rho^{*}, t\right] .
\end{array}\right.
$$

Then, for given $r_{t}=i \in \wp, P_{1 r_{t}}=P_{1 i}, P_{2 r_{t}}=P_{2 i}, P_{3 r_{t}}=P_{3 i}$ and the weak infinitesimal operator $L$ of the stochastic process $x(t)$ along the evolution of $V_{k}\left(x_{t}, t, r_{t}\right)(k=1,2, \cdots, N)$ are given as

$$
\begin{aligned}
& L V_{1}\left(x_{t}, t, i\right)=2\left[\begin{array}{ll}
x^{\mathrm{T}}(t) & \dot{x}^{\mathrm{T}}(t)
\end{array}\right]\left[\begin{array}{cc}
P_{1 i} & P_{2 i}^{\mathrm{T}} \\
0 & P_{3 i}^{\mathrm{T}}
\end{array}\right]\left[\begin{array}{c}
x(t) \\
0
\end{array}\right]+x^{\mathrm{T}}(t)\left[\sum_{j=1}^{N} \lambda_{i j} P_{1 j}\right] x(t) \\
& =2\left[\begin{array}{ll}
x^{\mathrm{T}}(t) & \dot{x}^{\mathrm{T}}(t)
\end{array}\right]\left[\begin{array}{cc}
P_{1 i} & P_{2 i}^{\mathrm{T}} \\
0 & P_{3 i}^{\mathrm{T}}
\end{array}\right]\left[\begin{array}{c}
x(t) \\
A_{i} x(t)-\dot{x}(t)+C \dot{x}(t-\tau)+B_{i} x(t-h(t))+D w(t)
\end{array}\right] \\
& +x^{\mathrm{T}}(t)\left[\sum_{j=1}^{N} \lambda_{i j} P_{1 j}\right] x(t) \\
& =x^{\mathrm{T}}(t)\left[P_{2 i}^{\mathrm{T}} A_{i}+A_{i}^{\mathrm{T}} P_{2 i}\right] x(t)+2 x^{\mathrm{T}}(t)\left[P_{1 i}-P_{2 i}^{\mathrm{T}}+A_{i}^{\mathrm{T}} P_{3 i}\right] \dot{x}(t) \\
& +2 x^{\mathrm{T}}(t) P_{2 i}^{\mathrm{T}} B_{i} x(t-h(t))+2 x^{\mathrm{T}}(t) P_{2 i}^{\mathrm{T}} C_{i} \dot{x}(t-\tau)+2 x^{\mathrm{T}}(t) P_{2 i}^{\mathrm{T}} D_{i} w(t) \\
& -\dot{x}^{\mathrm{T}}(t)\left[P_{3 i}^{\mathrm{T}}+P_{3 i}\right] \dot{x}(t)+2 \dot{x}^{\mathrm{T}}(t)\left[P_{3 i}^{\mathrm{T}} B_{i} x(t-h(t))+2 \dot{x}^{\mathrm{T}}(t) P_{3 i}^{\mathrm{T}} C_{i} x(t-\tau)\right. \\
& \left.+2 \dot{x}^{\mathrm{T}}(t) P_{3 i}^{\mathrm{T}} D_{i} w(t)+x^{\mathrm{T}}(t) \sum_{j=1}^{N} \lambda_{i j} P_{1 j}\right] x(t) .
\end{aligned}
$$

Taking into account the situation that the information of transition probabilities is not accessible completely, due to $\sum_{j=1}^{N} \lambda_{i j}=0 \quad(i \in \wp)$, the following equations hold for arbitrary appropriate matrices $W_{i}=W_{i}^{\mathrm{T}}$ are satisfied

$$
-x^{\mathrm{T}}(t) \sum_{j=1}^{N} \lambda_{i j} W_{i} x(t)=0, \forall i \in \wp .
$$

It is trivial to obtain the following equality:

$$
\begin{aligned}
& L V_{1}\left(x_{t}, t, i\right) \\
& =x^{\mathrm{T}}(t)\left[P_{2 i}^{\mathrm{T}} A_{i}+A_{i}^{\mathrm{T}} P_{2 i}\right] x(t)+2 x^{\mathrm{T}}(t)\left[P_{1 i}-P_{2 i}^{\mathrm{T}}+A_{i}^{\mathrm{T}} P_{3 i}\right] \dot{x}(t) \\
& +2 x^{\mathrm{T}}(t) P_{2 i}^{\mathrm{T}} B_{i} x(t-h(t))+2 x^{\mathrm{T}}(t) P_{2 i}^{\mathrm{T}} C_{i} \dot{x}(t-\tau)+2 x^{\mathrm{T}}(t) P_{2 i}^{\mathrm{T}} D_{i} w(t) \\
& -\dot{x}^{\mathrm{T}}(t)\left[P_{3 i}^{\mathrm{T}}+P_{3 i}\right] \dot{x}(t)+2 \dot{x}^{\mathrm{T}}(t) P_{3 i}^{\mathrm{T}} B_{i} x(t-h(t)) \\
& +2 \dot{x}^{\mathrm{T}}(t) P_{3 i} T C_{i} \dot{x}(t-\tau)+2 \dot{x}^{\mathrm{T}}(t) P_{3 i}^{\mathrm{T}} D_{i} w(t) \\
& +x^{\mathrm{T}}(t)\left[\sum_{j \in U_{k}}^{N} \lambda_{i j}\left(P_{1 j}-W_{j}\right)\right] x(t)+x^{\mathrm{T}}(t)\left[\sum_{j \in U_{u k}}^{N} \lambda_{i j}\left(P_{1 j}-W_{j}\right)\right] x(t), \\
& \quad L V_{2}\left(x_{t}, t, i\right) \\
& \quad=x^{\mathrm{T}}(t) Q x(t)-(1-\dot{h}(t)) \mathrm{e}^{-\alpha h(t)} x^{\mathrm{T}}(t-h(t)) Q x(t-h(t)) \\
& \quad-\alpha \int_{t-h(t)}^{t} \mathrm{e}^{\alpha(s-t)} x^{\mathrm{T}}(s) Q x(s) \mathrm{d} s \\
& \leq x^{\mathrm{T}}(t) Q x(t)-\left(1-\dot{h}_{M}\right) \mathrm{e}^{-\alpha h_{M}} x^{\mathrm{T}}(t-h(t)) Q x(t-h(t))-\alpha V_{2},
\end{aligned}
$$




$$
\begin{aligned}
& L V_{3}\left(x_{t}, t, i\right) \\
& =\dot{x}^{\mathrm{T}}(t) R \dot{x}(t)-\mathrm{e}^{-\alpha \tau} \dot{x}^{\mathrm{T}}(t-\tau) R \dot{x}(t-\tau)-\alpha \int_{t-\tau}^{t} \mathrm{e}^{\alpha(s-t)} \dot{x}^{\mathrm{T}}(s) R \dot{x}(s) \mathrm{d} s \\
& =\dot{x}^{\mathrm{T}}(t) R \dot{x}(t)-\mathrm{e}^{-\alpha \tau} \dot{x}^{\mathrm{T}}(t-\tau) R \dot{x}(t-\tau)-\alpha V_{3}, \\
& L V_{4}\left(x_{t}, t, i\right) \\
& =\tau h_{M}\left(h_{M}+\tau\right) \dot{x}^{\mathrm{T}}(t) S \dot{x}(t)-\tau h_{M} \int_{t-h_{M}-\tau}^{t} \mathrm{e}^{\alpha(s-t)} \dot{x}^{\mathrm{T}}(s) S \dot{x}(s) \mathrm{d} s-\alpha V_{4} \\
& \leq \tau h_{M}\left(h_{M}+\tau\right) \dot{x}^{\mathrm{T}}(t) S \dot{x}(t)-h_{M} \mathrm{e}^{-\alpha\left(h_{M}+\tau\right)}\left[\int_{t-h_{M}-\tau}^{t-h(t)} \dot{x}^{\mathrm{T}}(s) S \dot{x}(s) \mathrm{d} s\right. \\
& \left.+\int_{t-h(t)}^{t} \dot{x}^{T}(s) S \dot{x}(s) \mathrm{d} s\right]-\alpha V_{4} \\
& \leq \tau h_{M}\left(h_{M}+\tau\right) \dot{x}^{\mathrm{T}}(t) S \dot{x}(t)+h_{M} \mathrm{e}^{-\alpha\left(h_{M}+\tau\right)}\left[\begin{array}{c}
x\left(t-h_{M}-\tau\right) \\
x(t-h(t))
\end{array}\right]^{\mathrm{T}}\left[\begin{array}{cc}
-S & S \\
S & -S
\end{array}\right] \\
& \\
& +\left[\begin{array}{c}
x\left(t-h_{M}-\tau\right) \\
x(t-h(t))
\end{array}\right]-\tau \mathrm{e}^{-\alpha\left(h_{M}+\tau\right)}\left(\int_{t-h(t)}^{t} \dot{x}^{\mathrm{T}}(s) \mathrm{d} s\right) S \int_{t-h(t)}^{t} \dot{x}(s) \mathrm{d} s-\alpha V_{4} .
\end{aligned}
$$

Then substituting (13-16) into (12), we further have

$$
\begin{aligned}
& L\left(x_{t}, t, i\right)+\alpha V\left(x_{t}, t, i\right)-\frac{\alpha}{w_{m}} w^{\mathrm{T}}(t) w(t) \\
& \leq x^{\mathrm{T}}(t)\left[P_{2 i}^{\mathrm{T}} A_{i}+A_{i}^{\mathrm{T}} P_{2 i}+Q+\alpha P_{1 i}+\sum_{j \in U_{k}}^{N} \lambda_{i j}\left(P_{1 j}-W_{j}\right)\right] x(t) \\
& +2 x^{\mathrm{T}}(t)\left[P_{1 i}-P_{2 i}^{\mathrm{T}}+A_{i}^{\mathrm{T}} P_{3 i}\right] \dot{x}(t)+2 x^{\mathrm{T}}(t) P_{2 i}^{\mathrm{T}} B_{i} x(t-h(t)) \\
& +2 x^{\mathrm{T}}(t) P_{2 i}^{\mathrm{T}} C_{i} \dot{x}(t-\tau)+2 x^{\mathrm{T}}(t) P_{2 i}^{\mathrm{T}} D_{i} w(t) \\
& +2 \dot{x}^{\mathrm{T}}(t)\left[R+\tau h_{M}\left(h_{M}+\tau\right) S-P_{3 i}-P_{3 i}^{\mathrm{T}}\right] \dot{x}(t) \\
& +2 \dot{x}^{\mathrm{T}}(t) P_{3 i}^{\mathrm{T}} B_{i} x(t-h(t))+2 \dot{x}^{\mathrm{T}}(t) P_{3 i}^{\mathrm{T}} C_{i} \dot{x}(t-\tau)+2 \dot{x}^{\mathrm{T}}(t) P_{3 i}^{T} D_{i} w(t) \\
& -x^{\mathrm{T}}(t-h(t))\left[-\left(1-h_{d}\right) \mathrm{e}^{-\alpha h_{M}} Q-h_{M} \mathrm{e}^{-\alpha\left(h_{M}+\tau\right)} S\right] x(t-h(t)) \\
& +2 h_{M} x^{\mathrm{T}}(t-h(t)) \mathrm{e}^{-\alpha\left(h_{M}+\tau\right)} S x\left(t-h_{M}-\tau\right)-\mathrm{e}^{-\alpha \tau} \dot{x}^{\mathrm{T}}(t-\tau) R \dot{x}(t-\tau) \\
& -h_{M} \mathrm{e}^{-\alpha\left(h_{M}+\tau\right)} x^{\mathrm{T}}\left(t-h_{M}-\tau\right) S x\left(t-h_{M}-\tau\right) \\
& -\tau \mathrm{e}^{-\alpha\left(h_{M}+\tau\right)}\left(\int_{t-h(t)}^{t} \dot{x}^{\mathrm{T}}(s) \mathrm{d} s\right) S\left(\int_{t-h(t)}^{t} \dot{x}(s) \mathrm{d} s\right) \\
& -\frac{\alpha}{w_{m}} w^{\mathrm{T}}(t) w(t)+x^{\mathrm{T}}(t)\left[\sum_{j \in U_{u k}}^{N} \lambda_{i j} P_{1 j}\right] x(t) \\
& =\xi^{\mathrm{T}}(t) \Phi_{i} \xi(t)+x^{\mathrm{T}}(t)\left[\sum_{j \in U_{u k}}^{N} \lambda_{i j}\left(P_{1 j}-W_{j}\right)\right] x(t) .
\end{aligned}
$$

where $\Phi_{i}$ is the same as defined in the Theorem 1 and $\xi^{\mathrm{T}}(t)=\left[x^{\mathrm{T}}(t) \dot{x}^{\mathrm{T}}(t) x^{\mathrm{T}}(t-h(t)) \dot{x}^{\mathrm{T}}(t-\tau) x^{\mathrm{T}}\left(t-h_{M}-\tau\right) \int_{t-h(t)}^{t} \dot{x}(s) \mathrm{d} s^{\mathrm{T}} w^{\mathrm{T}}(t)\right]$.

Thus, from matrix inequalities (10)-(11), we get

$$
L V\left(x_{t}\right)+\alpha V\left(x_{t}\right)-\frac{\alpha}{w_{m}^{2}} w(t)^{\mathrm{T}} w(t) \leq 0
$$

which means, by Lemma 1 , that 
$V\left(x_{t}, t, i\right)=V_{1}\left(x_{t}, t, i\right)+V_{2}\left(x_{t}, t, i\right)+V_{3}\left(x_{t}, t, i\right)+V_{4}\left(x_{t}, t, i\right) \leq 1$, and this results in $V_{1}\left(x_{t}, t, i\right)=x^{\mathrm{T}}(t) P_{1 i} x(t) \leq 1$ for $\forall i \in \wp$, since $V_{2}\left(x_{t}, t, i\right)+V_{3}\left(x_{t}, t, i\right)+V_{4}\left(x_{t}, t, i\right) \geq 0$ from (11).

So the reachable set of the system (1) is bounded by ellipsoid $\mathfrak{I}\left(P_{1 i}, 1\right)$ ( $i \in \wp$ ) defined in (7), which implies that the reachable sets of the system (1) having the constraints (2) is bounded by a non-ellipsoid boundary $\bigcap_{i \in \wp} \mathfrak{I}\left(P_{1 i}, 1\right)$. This completes the proof.

Remark 1. It should be noted that the more the unknown elements there are in (4), the lower the maximum of time delay will be in Theorem 1. Actually, if all transition probabilities are unknown, the corresponding system can be viewed as a switched neutral Markovian jump system under arbitrary switching. Thus, the conditions obtained in Theorem 1 will cover the results for arbitrary switched neutral Markovian jump systems with disturbances. In that case, one can see the bounds of reachable sets in Theorem 1 become seriously conservative, for many constraints. Fortunately, we can use the Lyapunov functional method to analyze the bound of reachable sets for the neutral Markovian jump system under the assumption that all transition probabilities are not known.

For finding the bound of reachable sets for neutral Markovian jump systems with all transition probabilities are not known, one can construct the following Lyapunov functional

$$
\begin{aligned}
& V\left(x_{t}, t, r_{t}\right) \\
& =\left[\begin{array}{ll}
x^{\mathrm{T}}(t) & \dot{x}^{\mathrm{T}}(t)
\end{array}\right]\left[\begin{array}{ll}
I & 0 \\
0 & 0
\end{array}\right]\left[\begin{array}{rr}
P_{1} & 0 \\
P_{2} & P_{3}
\end{array}\right]\left[\begin{array}{c}
x(t) \\
\dot{x}(t)
\end{array}\right]+\int_{t-h(t)}^{t} \mathrm{e}^{\alpha(s-t)} x^{\mathrm{T}}(s) Q x(s) \mathrm{d} s \\
& +\tau h_{M} \int_{-h_{M}-\tau}^{0} \int_{t+\theta}^{t} \mathrm{e}^{\alpha(s-t)} \dot{x}^{\mathrm{T}}(s) S \dot{x}(s) \mathrm{d} s \mathrm{~d} \theta+\int_{t-\tau}^{t} \mathrm{e}^{\alpha(s-t)} \dot{x}^{\mathrm{T}}(s) R \dot{x}(s) \mathrm{d} s .
\end{aligned}
$$

Following a similar line as in proof of Theorem 1, we can obtain the following Theorem.

Theorem 2. Consider the Markov neutral system (1) with all elements unknown in transition rate matrix (4), if there exist symmetric matrices $P_{2}, P_{3}$, $P_{1}>0, Q>0, R>0, S>0$ and a scalar $\alpha>0$ satisfying the following matrix inequalities for $i=1,2, \cdots, N$

$$
\left[\begin{array}{ccccccc}
\Phi_{i 11} & \Phi_{i 12} & \Phi_{i 13} & P_{2 i}^{\mathrm{T}} C_{i} & 0 & 0 & P_{2 i}^{\mathrm{T}} D_{i} \\
* & \Phi_{i 22} & \Phi_{i 23} & P_{3 i}^{\mathrm{T}} C_{i} & 0 & 0 & P_{3 i}^{\mathrm{T}} D_{i} \\
* & * & \Phi_{i 33} & 0 & \Phi_{i 35} & 0 & 0 \\
* & * & * & -\mathrm{e}^{-\alpha \tau} R & 0 & 0 & 0 \\
* & * & * & * & \Phi_{i 55} & 0 & 0 \\
* & * & * & * & * & \Phi_{i 66} & 0 \\
* & * & * & * & * & * & -\frac{\alpha}{w_{m}} I
\end{array}\right]<0,
$$

where 


$$
\begin{aligned}
& \Phi_{i 11}=P_{2 i}^{\mathrm{T}} A_{i}+A_{i}^{\mathrm{T}} P_{2 i}+Q+\alpha P_{1 i}, \\
& \Phi_{i 12}=P_{1 i}-P_{2 i}^{\mathrm{T}}+A_{i}^{\mathrm{T}} P_{3 i}, \\
& \Phi_{i 13}=P_{2 i}^{\mathrm{T}} B_{i}, \\
& \Phi_{i 23}=P_{3 i}^{\mathrm{T}} B_{i}, \\
& \Phi_{i 22}=R+\tau h_{M}\left(h_{M}+\tau\right) S-P_{3 i}-P_{3 i}^{\mathrm{T}}, \\
& \Phi_{i 33}=-\left(1-h_{d}\right) \mathrm{e}^{-\alpha h_{M}} Q-h_{M} \mathrm{e}^{-\alpha\left(h_{M}+\tau\right)} S, \\
& \Phi_{i 35}=h_{M} \mathrm{e}^{-\alpha\left(h_{M}+\tau\right)} S, \\
& \Phi_{i 55}=-h_{M} \mathrm{e}^{-\alpha\left(h_{M}+\tau\right)} S, \\
& \Phi_{i 66}=-\tau \mathrm{e}^{-\alpha\left(h_{M}+\tau\right)} S .
\end{aligned}
$$

Then, the reachable sets of the system (1) having the constraints (2) is bounded by a non-ellipsoid boundary $\bigcap_{i \in \mathfrak{\wp}} \mathfrak{I}\left(P_{1 i}, 1\right)$, which $\mathfrak{I}\left(P_{1 i}\right)(i \in \wp)$ is defined in (7).

Remark 2. The solution for (8-10) or (18-20), if it exists, need not be unique. It is well-known [10] that the volume of $\Im\left(P_{1 i}, 1\right)$ defined in (7) is proportional to $\operatorname{det}\left(P_{1 i}\right)^{1 / 2}$, which means a square root of the determinant of $\mathfrak{I}\left(P_{1 i}, 1\right)$, so the minimization of $\ln \left(\operatorname{det}\left(P_{1 i}\right)^{1 / 2}\right)$ is the same as minimizing the volume of $\mathfrak{J}\left(P_{1 i}, 1\right)$. However, the minimization of $\ln \left(\operatorname{det}\left(P_{1 i}\right)^{1 / 2}\right)$ needs additional variables, so we use a simple approximation as that in [16] [17] [21]. That is, maximize $\delta$ subject to $\delta I \leq P_{1 i}$ which can be equivalent to the following optimization problem:

$$
\begin{aligned}
& \text { minimize } \bar{\delta}\left(\bar{\delta}=\frac{1}{\delta}\right) \\
& \text { s.t. }\left\{\begin{array}{l}
\text { (a) }\left[\begin{array}{cc}
\bar{\delta} I & I \\
I & P_{1 i}
\end{array}\right] \geq 0, i \in \wp, \\
\text { (b) }(8-10) \text { or }(18-20) .
\end{array}\right.
\end{aligned}
$$

Remark 3. The matrix inequalities in Theorem 1 and Theorem 2 contain only one non-convex scalar $\alpha>0$ (for given $h_{M}$ and $h_{d}$ ), and these become LMI by fixing the scalar $\alpha$. The feasibility check of a matrix inequality having only one non-convex scalar parameter is numerically tractable, and a local optimum value of $\alpha$ can be found by fminsearch.m.

\section{Numerical Examples}

In this section, a numerical example demonstrates the effectiveness of the approaches presented in this paper. Consider the neutral Markov jump systems with three operation modes whose state matrices are listed as follow:

$$
\begin{aligned}
& \dot{x}(t)-C_{\left(t, r_{t}\right)} \dot{x}(t-0.1)=A_{\left(t, r_{t}\right)} x(t)+B_{\left(t, r_{t}\right)} x(t-0.5)+D_{\left(t, r_{t}\right)} w(t), \\
& x\left(t_{0}+\theta\right)=\varphi(\theta), \forall \theta \in\left[-\rho^{*}, 0\right],
\end{aligned}
$$

where 


$$
\begin{aligned}
& A_{1}=\left[\begin{array}{cc}
-2 & -1 \\
0 & -2
\end{array}\right], A_{2}=\left[\begin{array}{cc}
-3 & 0 \\
0 & -2
\end{array}\right], A_{3}=\left[\begin{array}{cc}
-2 & 0 \\
-1 & -1.5
\end{array}\right], \\
& B_{1}=\left[\begin{array}{cc}
-1 & 0 \\
-1 & -2
\end{array}\right], B_{2}=\left[\begin{array}{cc}
-2 & 0 \\
-1 & -1
\end{array}\right], B_{3}=\left[\begin{array}{cc}
-2 & 0 \\
0 & -2
\end{array}\right], \\
& C_{1}=\left[\begin{array}{cc}
-1 & 0 \\
-1 & -2
\end{array}\right], C_{2}=\left[\begin{array}{cc}
-2 & 0 \\
-1 & -1
\end{array}\right], C_{3}=\left[\begin{array}{cc}
-2 & 0 \\
0 & -2
\end{array}\right], \\
& D_{1}=\left[\begin{array}{c}
-0.15 \\
0.15
\end{array}\right], D_{2}=\left[\begin{array}{c}
-0.14 \\
0.35
\end{array}\right], D_{3}=\left[\begin{array}{c}
-0.2 \\
0.3
\end{array}\right] .
\end{aligned}
$$

The transition rate matrix $\Lambda$ is considered as the following three cases.

Case 1. The transition rate matrix $\Lambda$ is completely known, which is considered as

$$
\Lambda=\left[\begin{array}{ccc}
-0.6 & 0.2 & 0.4 \\
0.6 & -1 & 0.4 \\
0.3 & 0.5 & -0.8
\end{array}\right]
$$

Case 2. The transition rate matrix $\Lambda$ is partly known, which is considered as

$$
\Lambda=\left[\begin{array}{ccc}
-0.6 & 0.2 & 0.4 \\
? & -1 & ? \\
? & ? & ?
\end{array}\right] .
$$

Case 3. The transition rate matrix $\Lambda$ is completely unknown, which is considered as

$$
\Lambda=\left[\begin{array}{lll}
? & ? & ? \\
? & ? & ? \\
? & ? & ?
\end{array}\right] .
$$

Firstly, by giving the transition probabilities $\Lambda$, a possible mode evolution of the neutral Markov jump system (22) is derived as shown in Figure 1. Based on the mode evolution shown in Figure 1, and choosing disturbances $w(t)$ as the random signal satisfying $w^{\mathrm{T}}(t) w(t) \leq 1$, all the reachable states of neutral Markov jump system (22) starting from the origin are given in Figure 2.

By using theorem 2 and solving the optimization problem (20) in case 1, we can obtain the maximize $\delta=0.61$ when $\alpha=0.1$, and the corresponding feasible matrices are given as $P_{11}=\left[\begin{array}{ll}7.2962 & 3.0026 \\ 3.0026 & 2.0132\end{array}\right], \quad P_{12}=\left[\begin{array}{ll}6.7864 & 2.6325 \\ 2.6325 & 1.7363\end{array}\right]$, $P_{13}=\left[\begin{array}{ll}6.4850 & 2.4905 \\ 2.4905 & 1.6669\end{array}\right]$. The reachable sets of the system (22) in case 1 is bounded by a intersection of three ellipsoids: $\bigcap_{i=1}^{3} \mathfrak{I}\left(P_{1 i}, 1\right)$, which is depicted in Figure 3.

By using theorem 1 and solving the optimization problem (22) in case 2, we can obtain the maximize $\delta=0.61$ when $\alpha=0.1$, and the corresponding feasible matrices are given as $P_{11}=\left[\begin{array}{ll}6.5942 & 2.5361 \\ 2.5361 & 1.6849\end{array}\right], \quad P_{12}=\left[\begin{array}{ll}6.5941 & 2.5362 \\ 2.5362 & 1.6849\end{array}\right]$, $P_{13}=\left[\begin{array}{ll}6.5954 & 2.5366 \\ 2.5366 & 1.6851\end{array}\right]$. The reachable sets of the system (22) in case 2 is bounded 
by a intersection of three ellipsoids: $\bigcap_{i=1}^{3} \Im\left(P_{1 i}, 1\right)$, which is depicted in Figure 4.

By using theorem 3 and solving the optimization problem (22) in case 3, we can obtain the maximize $\delta=0.65$ when $\alpha=0.1$, and the corresponding feasible matrices are given as $P_{11}=\left[\begin{array}{ll}8.1386 & 4.3002 \\ 4.3002 & 4.8168\end{array}\right], P_{12}=\left[\begin{array}{ll}9.0931 & 2.6563 \\ 2.6563 & 2.5718\end{array}\right]$, $P_{13}=\left[\begin{array}{ll}6.8607 & 2.6735 \\ 2.6735 & 1.8013\end{array}\right]$. The reachable sets of the system (22) in case 3 is bounded by a intersection of three ellipsoids: $\bigcap_{i=1}^{3} \Im\left(P_{1 i}, 1\right)$, which is depicted in Figure 5.

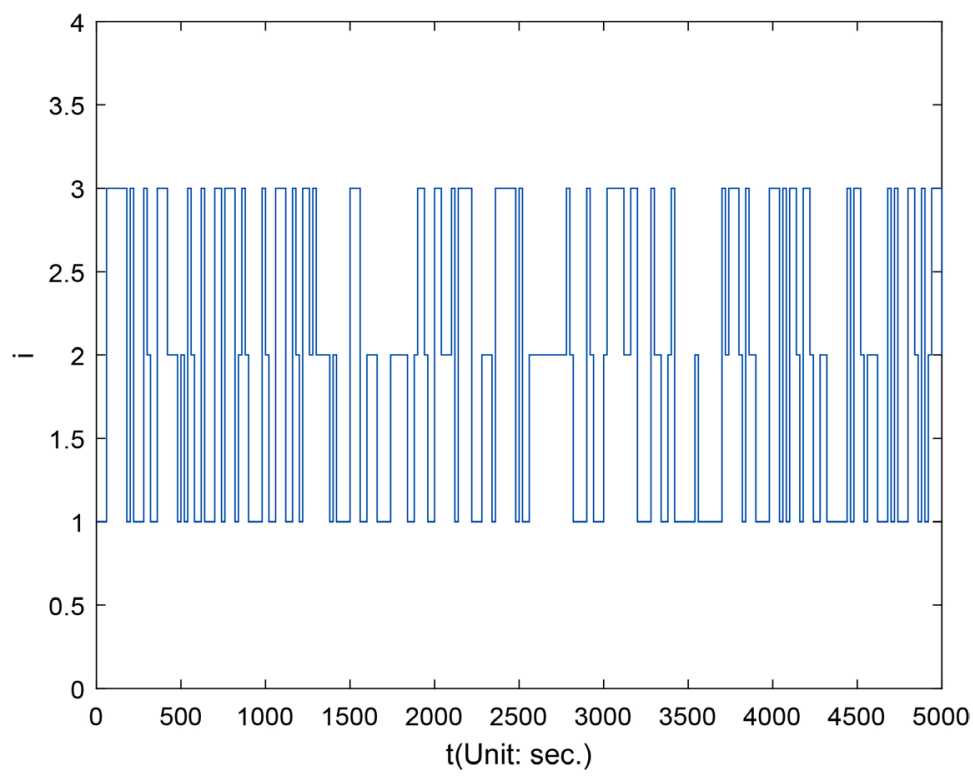

Figure 1. Random jumping mode $r(t)$ of neutral Markov jump system (22).
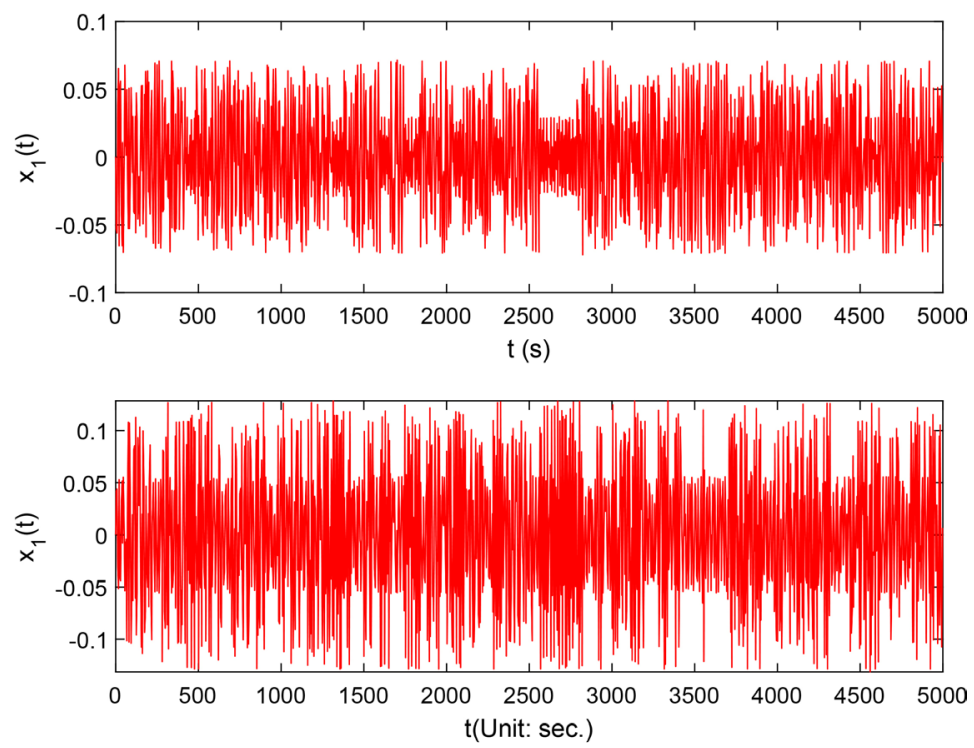

Figure 2. The time responses of state variable $x(t)$ of neutral Markov jump system (22) in case 1. 


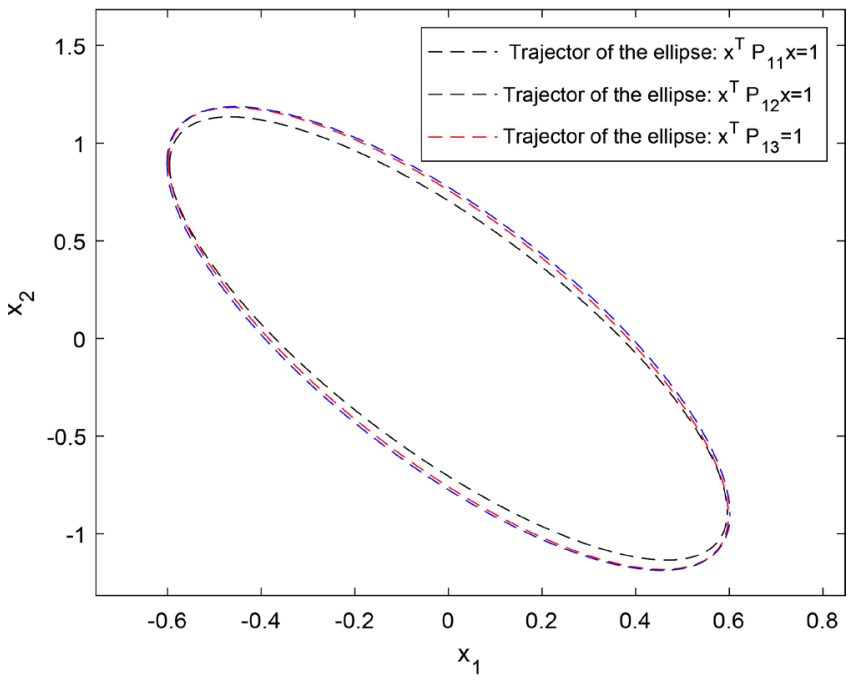

Figure 3. The bound of reachable sets for neutral Markov jump system (22) in case 1.

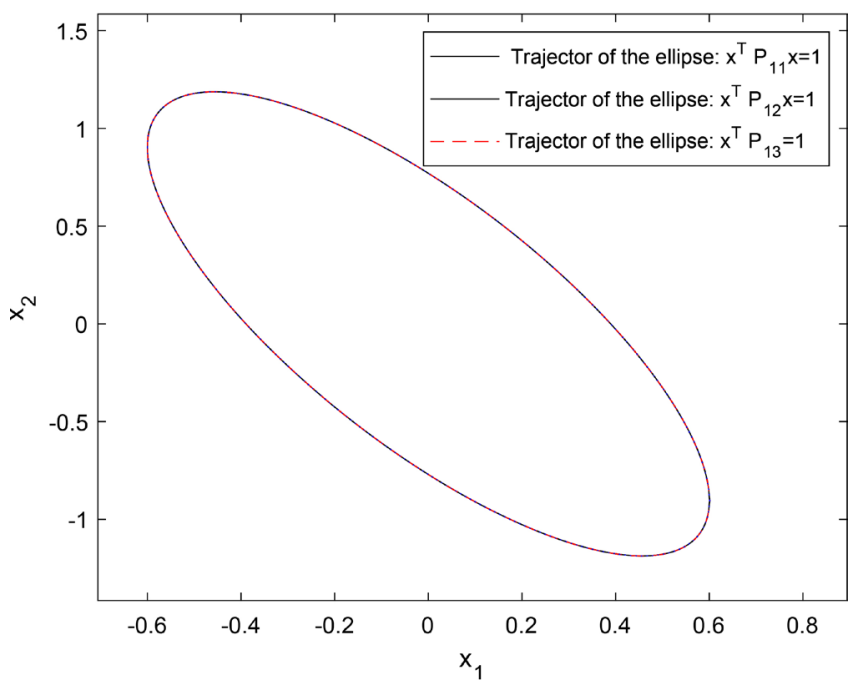

Figure 4. The bound of reachable sets for neutral Markov jump system (22) in case 2.

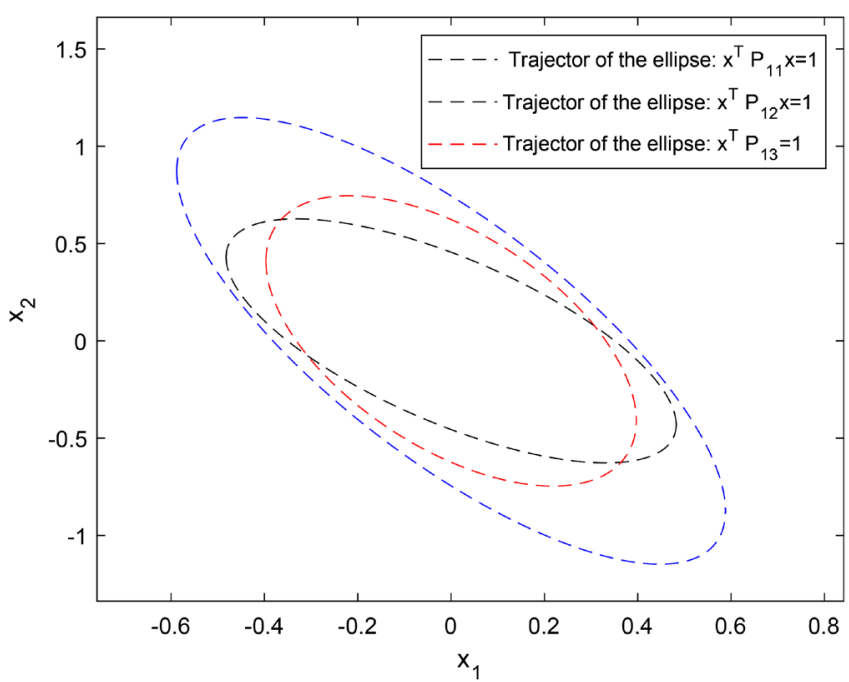

Figure 5. The bound of reachable sets for neutral Markov jump system (22) in case 3. 


\section{Conclusion}

In this paper, the problem of robust stability for a class of uncertain neutral systems with time-varying delays is investigated. Sufficient conditions are given in terms of linear matrix inequalities which can be easily solved by LMI Toolbox in Matlab. Numerical examples are given to indicate significant improvements over some existing results.

\section{Acknowledgements}

This research was supported by Science and Technology Foundation of Guizhou Province (No. LKM[2013]21; No. J[2015]2074; No. J[2016]1074), Innovation Group Major Program of Guizhou Province (No. KY[2016]029) and National Natural Science Foundation of China (No. 11761021).

\section{Conflicts of Interest}

The authors declare no conflicts of interest regarding the publication of this paper.

\section{References}

[1] Li, Z.-Y., Lam, J. and Wang, Y. (2018) Stability Analysis of Linear Stochastic Neutral-Type Time-Delay Systems with Two Delays. Automatica, 91, 179-189. https://doi.org/10.1016/j.automatica.2018.01.014

[2] Long, S.H., Zhong, S.M., Guan, H.B. and Zhang, D. (2019) Exponential Stability Analysis for a Class of Neutral Singular Markovian Jump Systems with Time-Varying Delays. Journal of the Franklin Institute, 356, 6015-6040. https://doi.org/10.1016/j.jfranklin.2019.04.036

[3] Qi, W.H., Yang, X., Gao, X.W., Cheng, J., Kao, Y.G. and Wei, Y.L. (2020) Stability for Delayed Switched Systems with Markov Jump Parameters and Generally Incomplete Transition Rates. Applied Mathematics and Computation, 365, Article ID: 124718. https://doi.org/10.1016/j.amc.2019.124718

[4] Wang, B. and Zhu, Q.X. (2020) The Novel Sufficient Conditions of Almost Sure Exponential Stability for Semi-Markov Jump Linear Systems. System and Control Letters, 137, Article ID: 104622. https://doi.org/10.1016/j.sysconle.2020.104622

[5] Hu, T. and Lin, Z. (2001) Control Systems with Actuator Saturation: Analysis and Design. Birkhauser, Boston. https://doi.org/10.1007/978-1-4612-0205-9

[6] Claudio, A. (2002) The Reachable Set of a Linear Endogenous Switching System. Systems \& Control Letters, 47, 343-353. https://doi.org/10.1016/S0167-6911(02)00228-1

[7] Collins, P. (2005) Continuity and Computability of Reachable Sets. Theoretical Computer Science, 341, 162-195. https://doi.org/10.1016/j.tcs.2005.05.001

[8] Wang, X.L., Xia, J.W., Wang, J., Wang, Z. and Wang, J. (2020) Reachable Set Estimation for Markov Jump LPV Systems with Time Delays. Applied Mathematics and Computation, 3761, Article ID: 125117. https://doi.org/10.1016/j.amc.2020.125117

[9] Wang, W.Q., Zhong, S.M., Liu, F. and Cheng, J. (2019) Reachable Set Estimation for Linear Systems with Time-Varying Delay and Polytopic Uncertainties. Journal of the Franklin Institute, 356, 7322-7346.

https://doi.org/10.1016/j.jfranklin.2019.03.031 
[10] Boyd, S., El Ghaoui, L., Feron, E. and Balakrishnan, V. (1994) Linear Matrix Inequalities in Systems and Control Theory. SIAM, Philadelphia. https://doi.org/10.1137/1.9781611970777

[11] Hu, T. and Lin, Z. (2003) Composite Quadratic Lyapunov Functions for Constrained Control Systems. IEEE Transactions on Automatic Control, 48, 440-450. https://doi.org/10.1109/TAC.2003.809149

[12] Kim, J.H. and Jabbari, F. (2004) Scheduled Controllers for Buildings under Seismic Excitation with Limited Actuator Capacity. Journal of Engineering Mechanics, 130, 800-808. https://doi.org/10.1061/(ASCE)0733-9399(2004)130:7(800)

[13] Kwon, O.M., Park, J.H. and Lee, S.M. (2008) On Stability Criteria for Uncertain Delay-Differential Systems of Neutral Type with Time-Varying Delays. Applied Mathematics and Computation, 197, 864-873.

https://doi.org/10.1016/j.amc.2007.08.048

[14] Wang, B., Liu, X. and Zhong, S. (2008) New Stability Analysis for Uncertain Neutral System with Time-Varying Delay. Applied Mathematics and Computation, 197, 457-465. https://doi.org/10.1016/j.amc.2007.07.034

[15] Zhang, L., Wang, C.H. and Gao, H.J. (2007) Delay-Dependent Stability and Stabilization of a Class of Linear Switched Time-Varying Delay Systems. Journal of Systems Engineering and Electronics, 18, 320-326. https://doi.org/10.1016/S1004-4132(07)60093-0

[16] Fridman, E. (2003) On Reachable Sets for Linear Systems with Delay and Bounded Peak Inputs. Automatica, 39, 2005-2010. https://doi.org/10.1016/S0005-1098(03)00204-8

[17] Kim, J.H. (2008) Improved Ellipsoidal Bound of Reachable Sets for Time-Delayed Linear Systems with Disturbances. Automatica, 44, 2940-2943. https://doi.org/10.1016/j.automatica.2008.03.015

[18] Hien, L.V. and Phat, V.N. (2009) Exponential Stability and Stabilization of a Class of Uncertain Linear Time-Delay Systems. Journal of the Franklin Institute, 346, 611-625. https://doi.org/10.1016/j.jfranklin.2009.03.001

[19] Lien, C.H., Yu, K.W, Chung, Y.J., Lin, Y.F., Chung, L.Y. and Chen, J.D. (2009) Exponential Stability Analysis for Uncertain Switched Neutral Systems with Interval-Time-Varying State Delay. Nonlinear Analysis. Hybrid Systems, 3, 334-342. https://doi.org/10.1016/j.nahs.2009.02.010

[20] Mondie, S. and Kharitonov, V.L. (2005) Exponential Estimates for Retarded Time-Delay Systems: An LMI Approach. IEEE Transactions on Automatic Control, 50, 268-273. https://doi.org/10.1109/TAC.2004.841916

[21] Zuo, Z.Q., Ho, D.W.C. and Wang, Y.J. (2010) Reachable Set Bounding for Delayed Systems with Polytopic Uncertainties: The Maximal Lyapunov-Krasovskii Functional Approach. Automatica, 46, 949-952. https://doi.org/10.1016/j.automatica.2010.02.022

[22] Li, M. and Liu, L. (2009) A Delay-Dependent Stability Criterion for Linear Neutral Delay Systems. Journal of the Franklin Institute, 346, 33-37. https://doi.org/10.1016/j.jfranklin.2008.06.001

[23] Qiu, F., Cui, B.T. and Ji, Y. (2010) Further Results on Robust Stability of Neutral System with Mixed Time-Varying Delays and Nonlinear Perturbations. Nonlinear Analysis. Real World Applications, 11, 895-906.

https://doi.org/10.1016/j.nonrwa.2009.01.032

[24] Shen, C.C. and Zhong, S.M. (2009) New Delay-Dependent Robust Stability Criterion for Uncertain Neutral Systems with Time-Varying Delay and Nonlinear Un- 
certainties. Chaos, Solitons \& Fractals, 40, 2277-2285.

https://doi.org/10.1016/j.chaos.2007.10.020

[25] Zhang, D. and Yu, L. (2010) $H_{\infty}$ Filtering for Linear Neutral Systems with Mixed Time-Varying Delays and Nonlinear Perturbations. Journal of the Franklin Institute, 347, 1374-1390. https://doi.org/10.1016/j.jfranklin.2010.05.001 